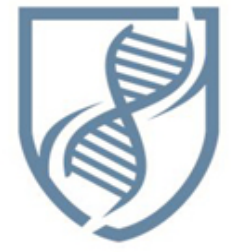

Journal of Bioscience and Applied Research
JBAAR

WWW.JBAAR.ORG

\title{
Effects of L-carnitine and Ginkgo bilobaon cerebral cortex in experimentally-induced epileptic seizures disease in rat
}

\author{
EhabTousson $^{1}$;Mohamed F.F Bayomy ${ }^{2}$ and BasiounyFouad El-sendiony ${ }^{2}$ \\ ${ }^{1}$ Zoology Department, Faculty of Science, Tanta University, Egypt. \\ ${ }^{2}$ Zoology Department, Faculty of Science, Menoufiya University, Egypt. \\ (*).corresponding author e. mail.toussonehab@yahoo.com
}

\begin{abstract}
Pentylenetetrazole (PTZ) kindling is an acknowledged model for epilepsy and refers to a phenomenon in which repeated injection of this drug causes gradual seizure. The current study aimed to determine the possible protective and ameliorative effects of L-carnitine and Ginkgo bilobaleaf extracts (GLE) against PTZinduced epileptic seizures disease in rats. A total of 80 male albino rats were equally divided into eight groups; $1^{\text {st }}$ group was the control; $2^{\text {nd }}$ and $3^{\text {rd }}$ wereGLE and L-carnitine groups, respectivel; while the $4^{\text {th }}$ group was pentylenetetrazolerat groupand the $5^{\text {th }}$ and $6^{\text {th }}$ groups were pre GLE and L-carnitine groups respectively; the $7^{\text {th }}$ and $8^{\text {th }}$ groups were post GLE and Lcarnitine, respectively. PTZ leads to an increase in malondialdehyde (MDA), glutamate (Glu) and to a decrease in catalase, super oxide dismutase (SOD), glutathione peroxidase (GPx) activity, glutathione reductase(GSH), gamma amino butyric acid (GABA) and acetylcholine esterase $(\mathrm{AChE})$ in the cerebral cortex of rats when compared to control group. Pre- and post treatment with GLE and L-carnitine were improved the biochemicalalterations in cerebral cortex that treated with PTZ.

excessive or synchronous neuronal activity in the brain (Fisher et al., 2005). A seizure is an event and epilepsy is the disease involving recurrent un- provoked seizures (Fisher et al., 2014).In 1981, the International League against Epilepsy (ILAE) developed an international classification of epileptic seizures that divides seizures into 2 major classes: partial-onset seizures and generalizedonset seizures. Partial-onset seizures begin in one focal area of the cerebral cortex, while generalized-onset seizures have an onset recorded simultaneously in both cerebral hemispheres. Some seizures are difficult to fit into a particular class, and they are considered as unclassified seizures (Commission on classification and terminology of the ILAE, 1981).

Pentylenetetrazole(PTZ) kindling is an acknowledged model for epilepsy and refers to a phenomenon in which repeated injection of a convulsant causes gradual seizure development culminating in generalized tonic-clonic seizures associated with a cognitive deficit (Sarkisian 2001). PTZ is considered a non-competitive gammaaminobutyric acid antagonist and has been used experimentally to study seizure phenomenon and to Keywords:Pentylenetetrazole; Epilepsy; Seizures; Cerebral identify pharmaceuticals that may control seizure cortex; Ginkgo biloba; L-carnitine; Oxidative stress; EZR-ir.

\section{Introduction}

susceptibility (Mandhane et al., 2007).

Oxidative stress is known to appear because of an imbalance between the production and degradation of Epilepsy is the neurological disorder of the human brain characterized by seizures affecting people of all ages, race, social class or geographical boundaries, affecting about 65 million personsworldwide, of whom up to $75 \%$ live in resource-poor countries(Hermann et al., 2012).Seizures and epilepsy are not the same. An epileptic seizure is a transient occurrence of signs and/or symptoms due to abnormal reactive oxygen species(ROS) in tissues. ROS-induced injury is well characterized and includes DNA base oxidation, lipid peroxidation, and protein oxidation (Laskinet al., 2010).In the last years, anumber of herbal products have been demonstrated to have promising anticonvulsant activity. These herbal products are candidates to be included in combination therapy of
\end{abstract}


epilepsy due to their considerable safety and lower side effects. Among these herbal products are Ginkgo biloba and L-carnitine(Samuels et al., 2008; Salama et al., 2013,;Tousson et al., 2014).Two main pharmacologically active groups compounds were present in ginkgoare the flavonoids and the terpenoids. The flavonoid is known to act mainly as antioxidants (Moreno et al., 2004; Tousson et al., 2014).

L-carnitine is naturally found at relatively high concentrations in certain foods such as red meat, beans, and avocado. However, depending upon diet, age, and other factors, supplementation of carnitine may be needed to maintain adequate levels in the body (Martinotti et al., 2011). In recent years, several antioxidants have shown the ability to protect neurons from ROS toxicity and degeneration. L-carnitine, among a wide array of target neuroprotective agents, is of particular interest in neurons due to its role in amino acid synthesis, ATP metabolism and mitochondrial fatty acid transport, as well as its antioxidant effects (Jones et al. 2010; Salamaet al., 2013; Tousson et al., 2014).Recent studies suggest that Lcarnitine may play an important role in oxidative/antioxidative balance and has an antiperoxidative effect on several tissues (Cayiret al., 2009; Pehlivan et al., 2009; Salama et al., 2012, 2013; Tousson et al., 2014). Therefore, the present paper aimed to study the protection and ameliorating role of Ginkgo bilobaleaf extract (GLE) and L-carnitine in the physiological, histopathological and immunohistochemical altrations in pentylenetetrazole induced epilepsy in rats.

\section{Materials and Methods}

\subsection{Animals}

The experiments were performed using 80 male albino rats (Rattusnorvigicus) weighing $120 \pm 10 \mathrm{~g}$ and of 7-8 week's age. The rats were kept in the laboratory for one week before the experimental work and maintained on a standard $\operatorname{diet}(20 \%$ casein, $15 \%$ corn oil, $55 \%$ corn starch, $5 \%$ salt mixture and 5\% vitaminzed starch; Egyptian Company of Oils and Soap Kafr-Elzayat Egypt) and water was available ad libitum. The temperature in the animal room was maintained at $23 \pm 2{ }^{\circ} \mathrm{C}$ with a relative humidity of $55 \pm 5 \%$. Light was on a 12:12 hr light - dark cycle. The experimental protocol was approved by Local Ethics Committee and Animals Research.

\subsection{Animal grouping:}

The rats were equally divided into eight groups (10 rats each). $1^{\text {st }}$ group was control group in which rats never received any treatment. $2^{\text {nd }}$ group was positive control rats (-ve control + L-carnitine (300 $\mathrm{mg} / \mathrm{kg}$ body weight) $)$ once per day every other day for 9 days according to Tousson et al. (2014). while $3^{\text {rd }}$ group was positive control rats (-ve control + GLE (100 mg/kg body weight))once per day every other day for 9 days according to Rodriguez de Turco et al. (1993). $4^{\text {th }}$ group was the experimental group that treated with pentylenetetrazol (PTZ; $40 \mathrm{mg} / / \mathrm{kg}$ body weight)once per day every other day for 9 days according to Akula et al. (2007); Dhir et al. (2007) and Waggas and
Al-Hasani (2010). $5^{\text {th group }}$ The rats were treated with PTZ $(40 \mathrm{mg} / \mathrm{kg}$ ) once every $48 \mathrm{~h}$ for 9 days after L-carnitine $(300 \mathrm{mg} / \mathrm{kg})$ treated once every $48 \mathrm{~h}$ for 9 days. $6^{\text {th }}$ groupThe rats were treated with PTZ $(40 \mathrm{mg} / \mathrm{kg})$ once every $48 \mathrm{~h}$ for 9 days after Ginkgo biloba $(100 \mathrm{mg} / \mathrm{kg})$ leaf extract treated once every $48 \mathrm{~h}$ for 9 days. $7^{\text {th }}$ group The rats were injected with PTZ (40 mg/kg) once every $48 \mathrm{~h}$ for 9 days then treated withL-carnitine $(300 \mathrm{mg} / \mathrm{kg}$ ) once every $48 \mathrm{~h}$ for 9 days.and $8^{\text {th }}$ groupThe rats were injected with PTZ $(40 \mathrm{mg} / \mathrm{kg}$ ) once every $48 \mathrm{~h}$ for 9 days then treated with Ginkgo biloba $(100 \mathrm{mg} / \mathrm{kg})$ leaf extract once every 48 h for 9 days.

For kindling a dose of $40 \mathrm{mg} / \mathrm{kg}$ body weight PTZ was injected intraperitoneal once every $48 \mathrm{~h}$. After each injection the convulsive behaviour was observed for 20 min. The resultant seizures were classified as follows:

Stage 0: no response

Stage 1: ear and facial twitching

Stage 2: myoclonic jerks without rearing

Stage 3: myoclonic jerks rearing

Stage 4: turn over into side position, clonic-tonic seizures

Stage 5: turn over into back position, generalized clonictonic seizures.

\section{Tissue Homogenate}

1. Prior to dissection, perfuse tissue with a PBS (phosphate buffered saline) solution, $\mathrm{pH}$ 7.4. containing $0.16 \mathrm{mg} / \mathrm{ml}$ heparin to remove any red blood cells and clots.

2. Homogenize the tissue in $5-10 \mathrm{ml}$ cold buffer ( $\mathrm{i}, \mathrm{e}, 50 \mathrm{mM}$ potassium phosphate, $\mathrm{pH}$ 7.4. 1 mM EDTA) per gram tissue.

3. Centrifuge at $4,000 \mathrm{rpm}$ for 15 minutes at 4 ${ }^{\circ} \mathrm{C}$.

4. Remove the supernatant for assay and store on ice. If not assaying on the same day, freeze the sample at $-80^{\circ} \mathrm{C}$. The sample will be stable for at least one month.

\section{2,3 Enzymatic and non-enzymatic antioxidant assays} Malondialdehyde(MDA) assay:MDAis a noxious product of lipid peroxidation. It was detected by TBARS analysis and measured as reported by Satoh, (1978) and Ohkawa et al.(1979). The MDA results were expressed as the nmol/g tissue.

Reduced glutathione(GSH): GSH content was determined with dithionitrobenzoic acid using the method described by Beutler et al. (1963) and was expressed as mol GSH/mg protein. The method is based on the reduction of DTNB to produce a yellow compound. The reduced chromogen is directly proportional to GSH concentration and its absorbance can be measured at 412 $\mathrm{nm}$.

GSH-Px: GSH-Px content was determined using the method described by Paglia and Valentine (1967) and was expressed as U/g wet tissue. The method is based on oxidation of NADPH to NADP+ and is accompanied by a decrease in absorbance at $340 \mathrm{~nm}$ (A340) providing a spectrophotometric means for monitoring GPx enzyme activity. The molar extinction coefficient for NADPH is 
$6220 \mathrm{M}-1 \mathrm{~cm}-1$ at $340 \mathrm{~nm}$. To assay c-GPx, a cell or tissue homogenate is added to a solution containing glutathione, glutathione reductase, and NADPH. The enzyme reaction is initiated by adding the substrate, hydrogen peroxide and the A340 is recorded. The rate of decrease in the A340 is directly proportional to the GPx activity in the sample.

Superoxide dismutase (SOD): SODassay relies on the ability of the enzyme to inhibit the phenazinemethosulphate-mediated reduction of nitrobluetetrazolium dye at $560 \mathrm{~nm}$ according to the methods of Nishikimi (1972). The SOD results were expressed as the U/g wet tissue.

Catalase: The catalase (CAT) activity was measured by monitoring $\mathrm{H}_{2} \mathrm{O}_{2}$ (The substrate of the enzyme) decomposition at $240 \mathrm{~nm}$ according to the method described by Aebi (1984)and Fossati et al. (1980).

Acetyl cholinesterase (AChE): Acetylcholine esterase hydrolyses acetylcholine to give thiocholine and acetate.The reaction between thiocholine and DTNB gives 2-nitro-5 mercaptobenzoate, a yellow compound which can be measured at $405 \mathrm{~nm}$. according to Knedel and Bottger(1967).

Gamma aminobutyric acid (GABA): GABA concentrations in the brain homogenate were determined by pre-column derivatization with ophthaldialdehyde/mercaptoethanol reagent, and separation by reverse-phase HPLC in conjunction with fluorometric detection according to Lindroth and Mopper (1979). Results are shown as fmol/lL/mm.

Glutamate in brain homogenate:Chromatograms were compared to that of standard. Amount of neurotransmitter was estimated as the method reported by Shankaranarayana et al. (1998).

\subsection{Histopathological investigation}

Immediately after decapitation, animals were dissected, cerebral cortex from animals of different groups were quickly removed, washed in $0.9 \%$ saline solutions and samples of cerebral cortex were fixed in $10 \%$ neutral buffered formalin. After fixation, specimens were dehydrated in an ascending series of alcohol, cleared in two changes of xylene and embedded in molten paraffin (mp. $50-58^{\circ} \mathrm{C}$ ). Sections of 7 microns thickness were cut using rotary microtome and mounted on clean slides. Sections were stained with Ehrlich's haematoxylin and counterstained with eosin as a routine method by Bancroft and Stevens (1990).

2.5 Immunohistochemical detection of Ezrin (EZR-ir) Expression of EZR immunoreactivities (EZR -ir) was detected using avidin Biotin Complex (ABC) method (Helander et al., 1996). Paraffin sections (5 m thick) of fixed rat cerebral cortex that mounted on gelatin chromalum-coated glass slides were dewaxed and rehydrated sections were washed in distilled water for 5 min, rinsed in PBST for $10 \mathrm{~min}$ and incubated with $10 \%$ normal goat serum for $15 \mathrm{~min}$ to reduce non-specific background staining. Then, the sections were incubated with anti-rabbit EZR for 1-2 hours at room temperature. The sections after 5 baths in PBST were incubated with biotinylated goat antirabbitimmuoglobulin (Nichirei, Tokyo, Japan). The sections after 5 baths in PBST were further incubated with Avidin Biotin Complex (ABC: Nichirei, Tokyo, Japan) for 1 hour at RT. The reaction was developed using $20 \mathrm{mg}$ 3-3'-diaminobenzidine tetrahydrochloride (DAB, Wako pure chemical industries, Ltd) in $40 \mathrm{ml} \mathrm{PBST}$, pH 7.2 containing $10 \mathrm{ml}$ of hydrogen peroxide $(\mathrm{H} 2 \mathrm{O} 2)$ for $7-9 \mathrm{~min}$ at a dark room followed by distilled water then dehydrated and mounted. The criterion for a positive reaction confirming the presence of EZR is a dark, brownish, intra cytoplasmic precipitate. For the negative control, the primary antibody was omitted to guard against any false positive results which might develop from a non-specific reaction. Brightness, contrast were adjusted using Adobe Photoshop software. Image analysis was adjusted using PAX-it image analysis software.

\section{Statistical Analysis:}

Data were expressed as mean values \pm SEM and statistical analyses were performed using SPSS statistical version 16 software package (SPSS ${ }^{\circ}$ Inc., USA). The criterion for statistical significance was set at $\mathrm{p}<0.01$.

\section{Results}

Figures (1-8) show that both MDA levels and Glu in Pentylenetetrazole group (G4) showed significant increase when compared with control (G1), L-carnitine (G2) and GLE (G3) groups. On the other hand, catalase, superoxide dismutase (SOD), glutathione peroxidase activity (GPX), glutathione reductase (GSH), gamma amino butyric acid (GABA) and acetylcholinesterase (AChE) levels in pentylenetetrazole group (G4) showed significant decreases when compared with control (G1), GLE (G2) and Lcarnitine(G3) groups. In contrast, MDA levels in pre treated PTZ group (G4) with L-carnitine and GLEgroups (G5\&G6) were significantly decreased when compared with PTZ group (G4) while catalase, SOD, GPX, GSH, GABA and AChE levels in pre treated PTZ group (G4) with L-carnitine and GLE groups (G5\&G6) were significantly increased in pre treatedPTZ group (G4) with L-carnitine and GLE groups (G5\&G6) when compared withPTZ group (G4). MDA levels in post treated PTZ group (G4) with L-carnitine and GLE groups (G7\&G8) were significantly decreased when compared withPTZ group (G4) while Catalase, SOD, GPX, GSH, GABA and AChE levels in post treatedPTZ group (G4) with Lcarnitine and GLE groups (G7\&G8) were significantly increased in post treated PTZ group (G4) with L-carnitine and GLE groups (G7\&G8) when compared with PTZ group (G4).

\section{Histopathological findings in the brain cortex}

Representative coronal sections of H\&E stain obtained from the male rat brain cortex in different groups under study are shown at the light microscope levels in Figures (9 A - 9H). 


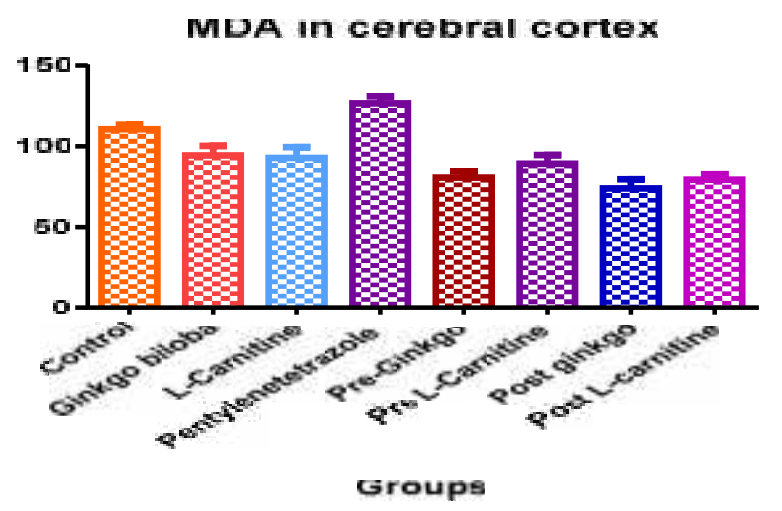

Fig.(1): Data are expressed as mean \pm SE. Significant difference from the control group (G1) at $P<0.05$. significant different from PTZ group (G4) at $\mathrm{P}<0$.05.MDA concentration in cerebral cortex homogenate (nmol/g tissue). MDA levels in PTZ group showed significant increase when compared with control group. In contrast, MDA levels in pre and post treated PTZ group with L-carnitine and GLE groups were significantly decreased when compared with PTZ group.

\section{GSH in cerebral cortex}

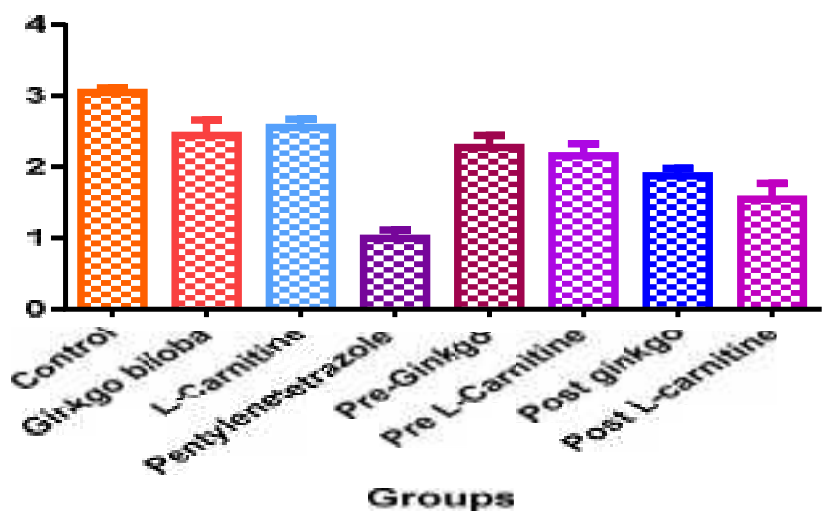

Fig.(2): Data are expressed as mean \pm SE. Significant difference from the control group (G1) at $P<0.05$. significant different from PTZ group (G4) at $\mathrm{P}<0.05$. Glutathione reductase concentration in cerebral cortex (mM).GSH levels in PTZ group showed significant decrease when compared with control group. In contrast, GSH levels in pre and post treated PTZ group with L-carnitine and GLE groups were significantly increased when compared with PTZ group.

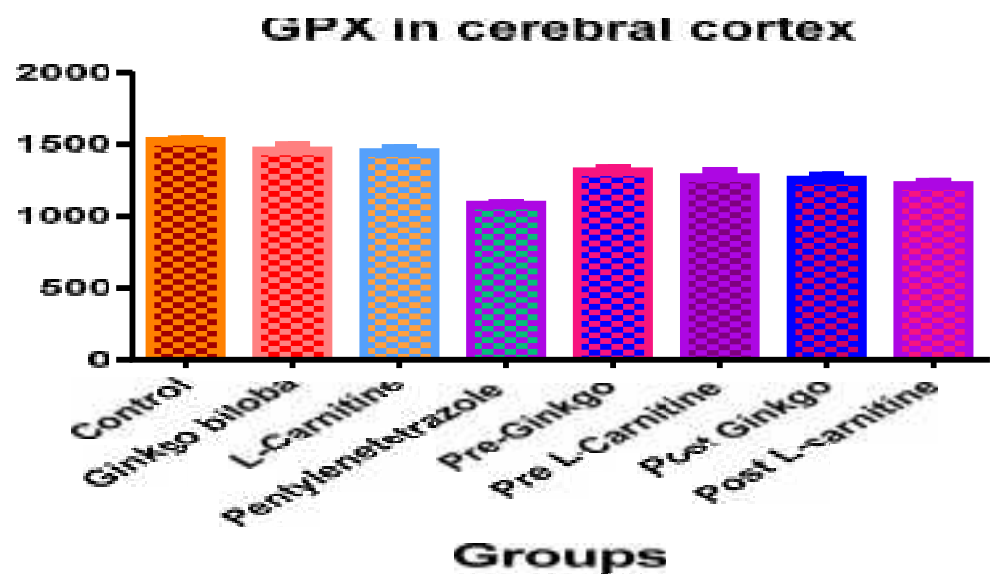

Fig.(3):Data are expressed as mean \pm SE. Significant difference from the control group (G1) at $P<0.05$. significant different from PTZ group (G4) at P<0.05.Glutathione peroxidase activity in cerebral cortex (U/gT).GPx levels in PTZ group showed significant decrease when compared with control group. In contrast, GPx levels in pre and post treated PTZ group with L-carnitine and GLE groups were significantly increased when compared with PTZ group. 


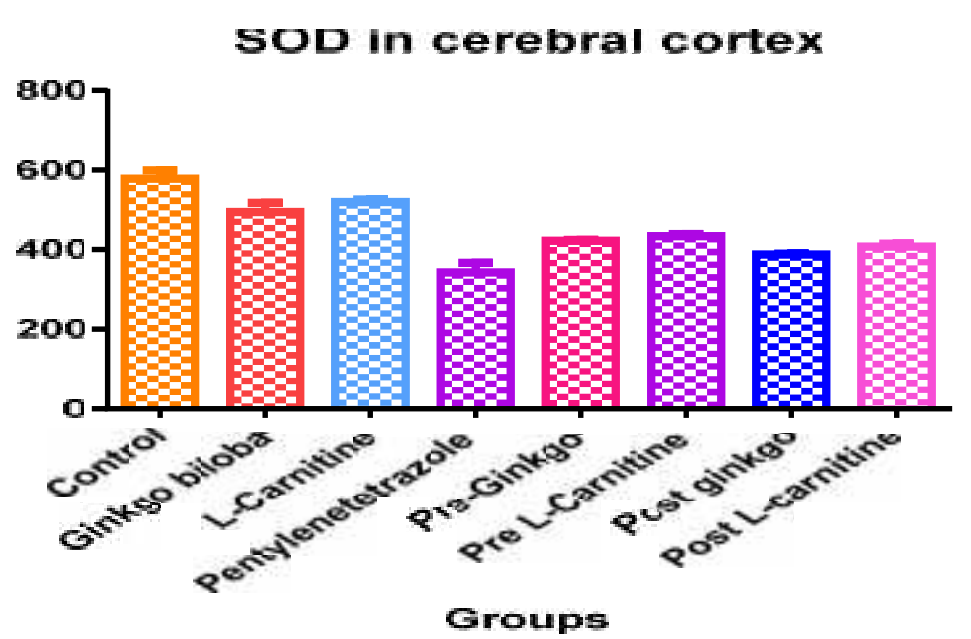

Fig.(4): Data are expressed as mean \pm SE. Significant difference from the control group (G1) at $P<0.05$. significant different from PTZ group (G4) at $\mathrm{P}<0$.05. Superoxide dismutaseactivity in cerebral cortex (U/gm tissue). SOD levels in PTZ group showed significant decrease when compared with control group. In contrast, SOD levels in pre and post treated PTZ group with L-carnitine and GLE groups were significantly increased when compared with PTZ group.

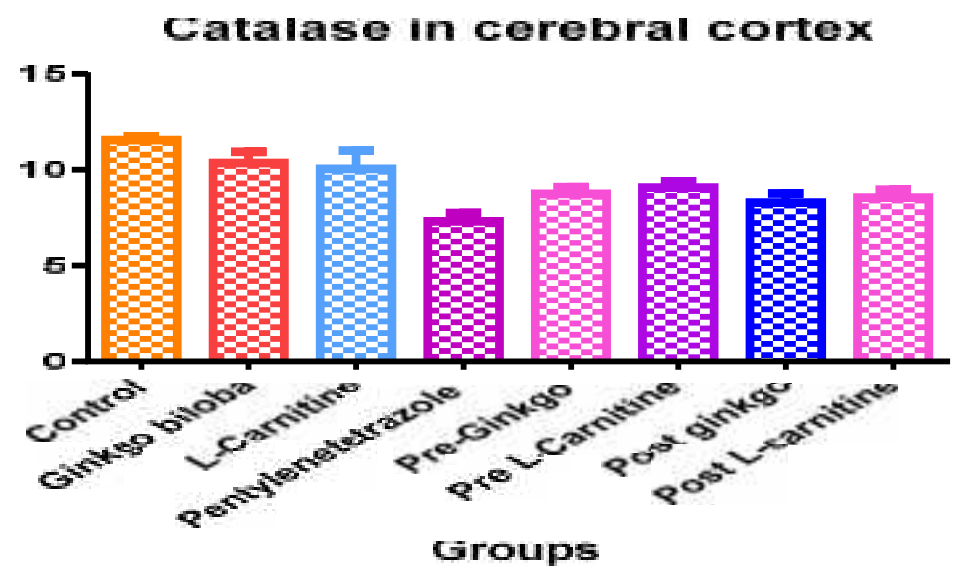

Fig.(5): Data are expressed as mean \pm SE. Significant difference from the control group (G1) at $\mathrm{P}<0.05$. significant different from PTZ group (G4) at $\mathrm{P}<0.05$.Catalase activity in cerebral cortex (U/g). Catalase levels in PTZ group showed significant decrease when compared with control group. In contrast, catalase levels in pre and post treated PTZ group with L-carnitine and GLE groups were significantly increased when compared with PTZ group.

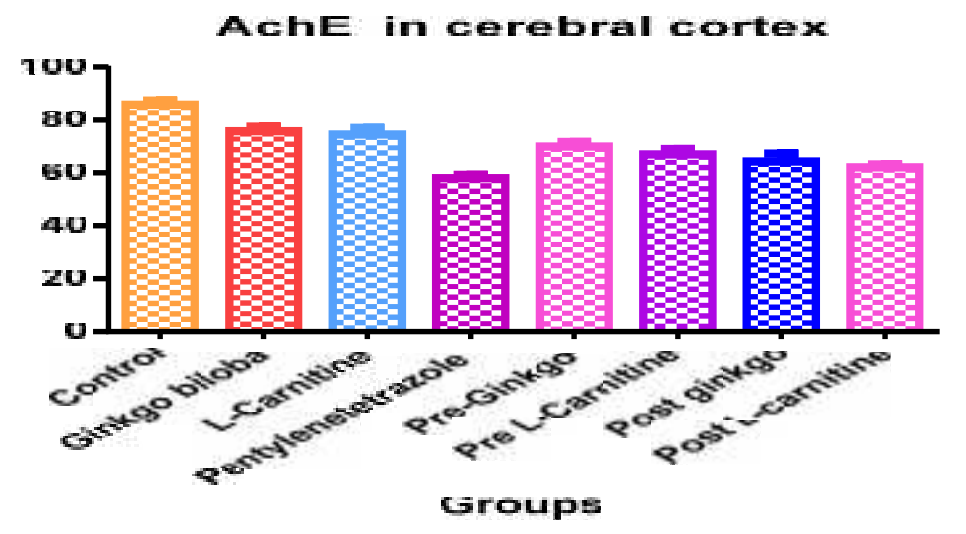

Fig.(6): Data are expressed as mean \pm SE. Significant difference from the control group (G1) at $\mathrm{P}<0.05$. significant different from PTZ group (G4) at $\mathrm{P}<0.05$. Acetylcholinesteraseactivity in cerebral cortex (U/L). AchE levels in PTZ group showed significant decrease when compared with control group. In contrast, AchE levels in pre and post treated PTZ group with L-carnitine and GLE groups were significantly increased when compared with PTZ group. 


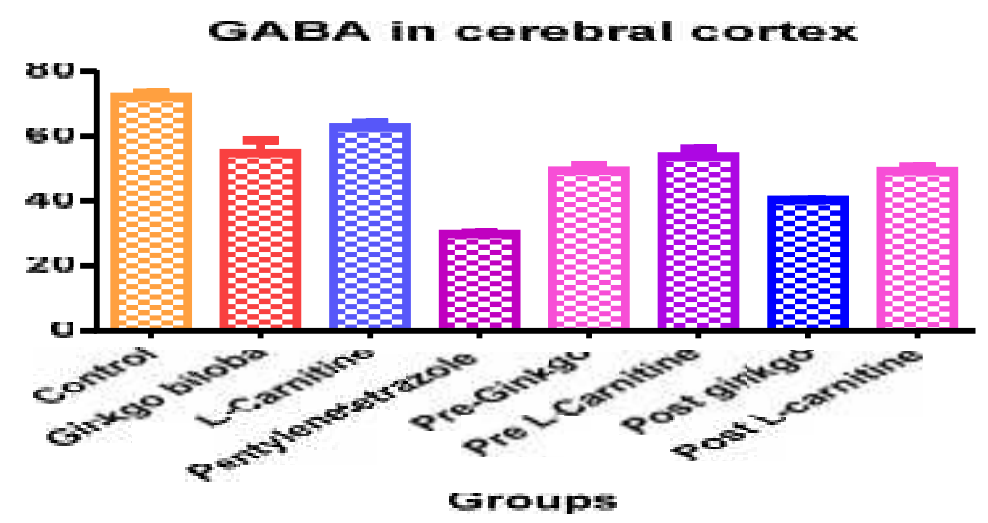

Fig.(7): Data are expressed as mean \pm SE. Significant difference from the control group $(\mathrm{G} 1)$ at $\mathrm{P}<0.05$. significant different from $\mathrm{PTZ}$ group (G4) at $\mathrm{P}<0.05$.Gammaminobytaric acid concentration in cerebral cortex (fmol/lL/mm). GABA levels in PTZ group showed significant decrease when compared with control group. In contrast, GABA levels in pre and post treated PTZ group with L-carnitine and GLE groups were significantly increased when compared with PTZ group.

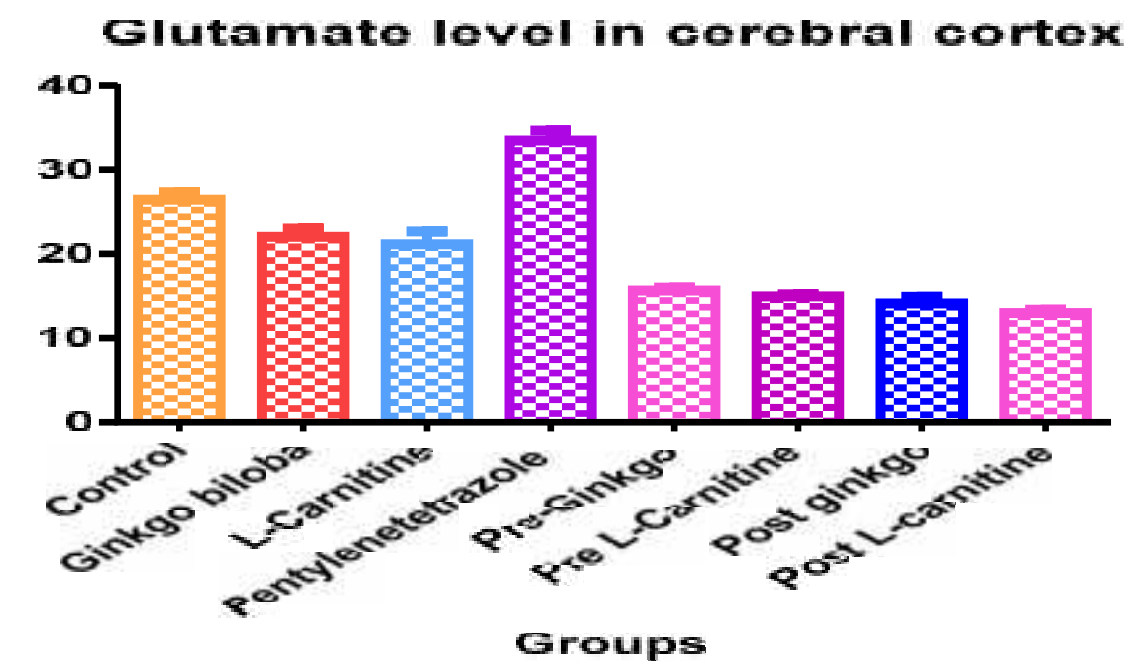

Fig.(8): Data are expressed as mean \pm SE. Significant difference from the control group $(\mathrm{G} 1)$ at $\mathrm{P}<0.05$. significant different from $\mathrm{PTZ}$ group (G4) at $\mathrm{P}<0.05$. Glutamateconcentration in cerebral cortex $(\mathrm{fmol} / \mathrm{lL} / \mathrm{mm})$. Glu levels in $\mathrm{PTZ}$ group showed significant increase when compared with control group. In contrast, Glu levels in pre and post treated PTZ group with L-carnitine and GLE groups were significantly decreased when compared with PTZ group.

The histopathological examination of PTZ rat brain cortex revealed various histopathological changes, as the presence of a large number of damaged neurons, diffuse vacuolar degeneration, moderate degenerating neurons and neuronal atrophy were observed in the rat brain cortex when compared with those of the control rats (Fig. 9D). Cortex sections in pro-treated PTZ with L-canitin revealed a good degree of improvement with more or less normal neuronal structure while cortex sections in pro-treated PTZ with GLE revealed mild to moderate neurofibrillary degeneration damaged neurons (Figs. 9E-9F). Cortex sections in post treated PTZ with L-canitin, respectively, revealed a few numbers of damagedneurons with mild atrophy and diffuse vacuolar degeneration (Figs. 9G).
Cortex sections in post treated PTZ with GLE revealed mild neuronal damage and atrophy $(9 \mathrm{H})$.

\section{Immunohistochemistry findings in the brain cortex}

The detection and distribution of Ezrinimmunoreactivity (ERZ-ir) are shown on neurons in the brain cortex in the different groups under study (Figures 10A-10H). Negative or Faint positive ERZ-ir neurons were not detected in the hippocampus of control (G1), L-carnitine (G2) and GLE (G3) groups (Figs. 10A-10C).

In PTZ rat group (G4), moderate ERZ positive neurons were detected in the cortex (Figs.10D). 
Cortex coronal sections in both pro-treated PTZ with Lcarnitine (G5) and with GLE (G6) revealed faint and mild positive reaction for ERZ-ir, respectively, (Figures 10E10F). The intensity of ERZ-ir neurons on cortex sections in pro-treated PTZ with GLE were increased when compared with pro-treated PTZ with L-carnitine.

Cortex coronal sections in both pro-treated PTZ with Lcarnitine (G5) and with GLE (G6) revealed faint and mild positive reaction for ERZ-ir, respectively, (Figures 10E10F). The intensity of ERZ-ir neurons on cortex sections in pro-treated PTZ with GLE were increased when compared with pro-treated PTZ with L-carnitine.

Cortex coronal sections in both post-treated PTZ with Lcarnitine (G7) and with GLE (G8) revealed mild to moderate reaction for ERZ-ir (Figures 10G-10H). The intensity of ERZ-ir neurons on cortex sections in posttreated PTZ with GLE were increased when compared with pro-treated PTZ with L-carnitine. The intensity of ERZ-ir neurons was depressed in pro- treated PTZ with Lcarnitineor GLE when compared with post-treated PTZ with L-carnitine or GLE (Figs.10G-10H).

\section{Discussion}

Epilepsy is one of the most common neurologic problems all over the world, being associated with paroxysmal discharge of cerebral neurons and is characterized by several symptoms including alterations of behaviors and consciousness (Godlevskii et al., 2004).

Pentylenetetrazole, a GABA receptor antagonist, is a wellestablished animal model to assess efficacy of drugs against generalized absence seizures in humans (Liuet al.(2012).Pentylenetetrazole-induced seizure activity mimics the increased oxidative stress in the brain by altering membrane phospholipid metabolism and ultimately resulting in the release of free radicals (Corvino et al.,2013).

L-Carnitineis a naturally occurring endogenous compound in all mammalian species and the most widely known function is its role as an important transporter of long chain fatty acids into mitochondria for $\beta$-oxidation (Bieber, 1988). L-carnitine, among a wide array of target neuroprotective agents, is of particular interest in neurons due to its role in amino acid synthesis, ATP metabolism, mitochondrial fatty acid transport, as well as its antioxidant effects (Gu“lc, in 2006; Jones et al. 2010; Nalecz et al. 2004; Silva-Adaya et al. 2008; Wu et al. 2011).

EGb761 is a standardized extract of GB leaves and has antioxidant properties as a free radical scavenger (ADIS and Profile, 2003). EGb761 decreases tissue levels of reactive oxygen species, inhibits membrane lipid peroxidation and, with its anti-platelet-activating factor activity, contributes to improvements in cerebral insufficiency (Liu et al., 2006).

This study conducts a biochemical investigation into whether L-carnitine and Ginkgo biloba have a protective and ameliorative effect on pentylenetetrazole-induced epilepsy in male rats. The results of the studies indicate that pentylenetetrazole causes oxidative tissue damage by increasing lipid peroxidation in the cerebral cortex tissues and decreasing the level of antioxidant enzymes.

In the present study, PTZ administration in rats over a period of 9 injection resulted in progressive seizure intensity (tonic-clonic), increased mortality rate and alteration in animal behavior. A significant rise in MDA levels was observed after PTZ administration in homogenate of rat cerebral cortex when compared to control animals.Similarly, Kutluhan et al. (2009) have reported increased levels of MDA in brain tissues in a mouse-seizure model after PTZ administration. A significant reduction in MDA levels was observed in rat cerebral cortex when treated with L-carnitine. This reduction is possibly due to the modulatory activity of Lcarnitine in the antioxidant enzymes in the brain of adult rats. This indicates that L-carnitineinhibitsneuronal damage as free radical scavenger.Similarly, Ahmed andMahmoud (2012) have reported that L-carnitine administration has decreased levels of MDA in brain tissues in a mouseseizure model.

Treatment with pre and post GB significantly reduced MDA level.Abdel-Wahab and Metwally(2011) have reported decreased levels of MDA as effects of GB of inhibition of lipid peroxidation. The present findings indicate that the effects of GB against oxidative damage may be due to its antioxidant and free radical-scavenging activity.

The present study demonstrated that A significant decrease in GSH reductase levels was observed after PTZ administration. Previous study was reported, PTZ induced seizure shows marked reduction of antioxidant enzymes like glutathione reductase(Patsoukis et al., 2004; Nieoczym, et al., 2008).

In this study, administration of pre and post L-carnitine induced significant increase in the GSH. This result is in agreement with the study made by Heba and Sameh (2012)who found that administration of L-carnitine induced significant increase in GSH content and this might be the active role of L-carnitine in protection against oxidative stress.

Pre and Post GLE treatments led to increasing levels of GSH in homogenate tissue when compared to PTZ group. These results are similar to othersobserved in astudy made by Pinakini et al.(2005).Ginkgo biloba, a known plant with its antioxidant property, probably strengthened the antioxidant status as evidenced by the observed increase in the GSH levels.

In the present study, the result demonstrating significant decrease in GPX levels was observed after PTZ administration. SekarBabu et al. (2011) documented that changes in glutathione peroxidase activity in brain homogenates were inversely correlated with intensity of lipid peroxidation. It may be supposed that decrease in glutathione peroxidase activity causes failure of $\mathrm{H}_{2} \mathrm{O}_{2}$ detoxification. 

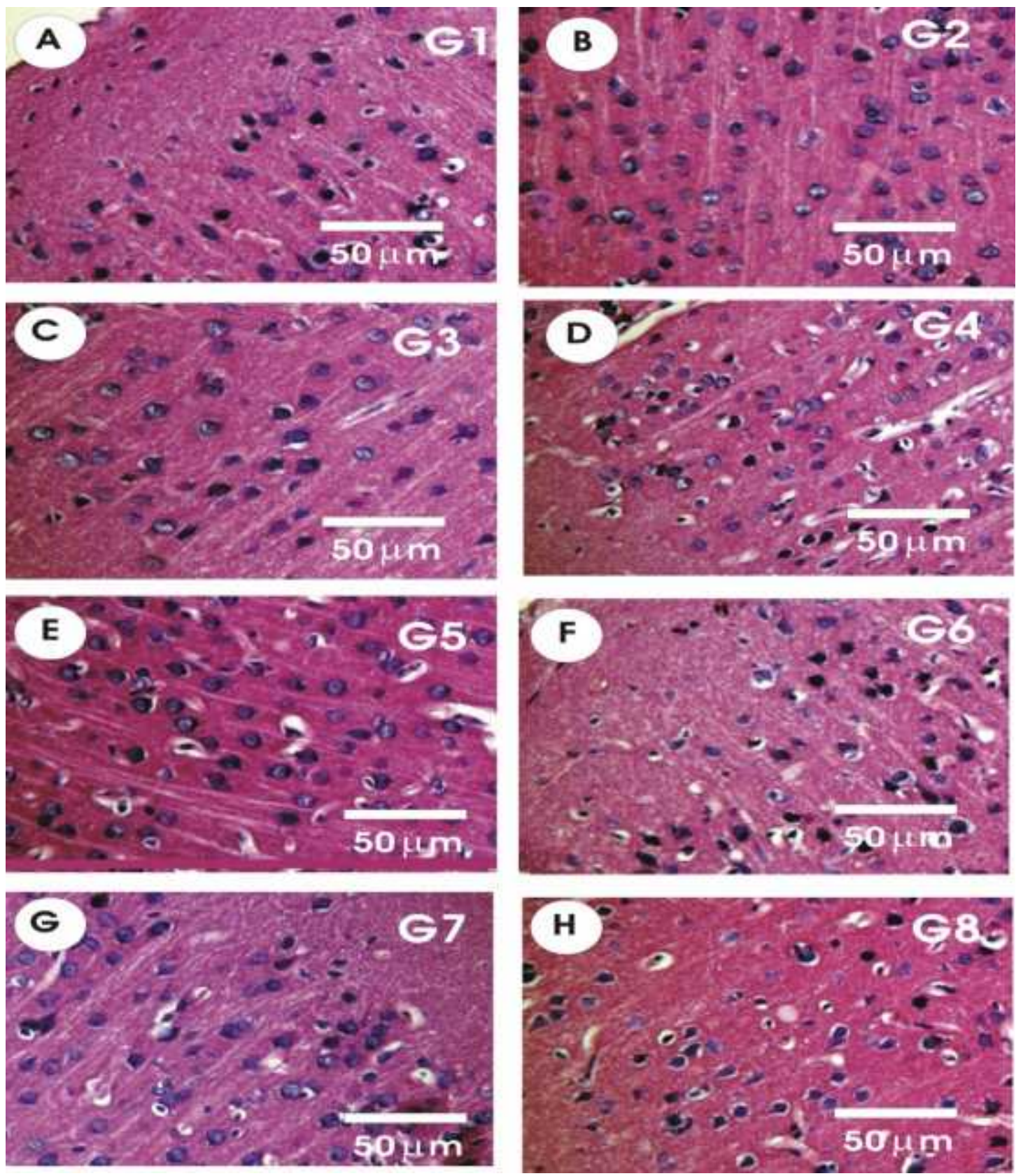

Figures 9A-9H: Photomicrographs of rat cortex sections in the different experimental groups stained with Haematoxylin\& Eosin. A-C: Rat cortex sections in control $\left(\mathrm{G}_{1}\right)$; L-carnitin $\left(\mathrm{G}_{2}\right)$ and GLE $(\mathrm{G} 3)$ groups revealed a normal neuronal structure. Observations of the rat cortex compared with the controlrats. D: Ratcortex sections of PTZ group $\left(\mathrm{G}_{4}\right)$ revealed marked damages in a large number of neurons, atrophy, diffuse vacuolar degeneration, moderate degenerating neurons. E: Cortex sections in pro-treated PTZ with L-canitin revealed a good degree of improvement with more or less normal neuronal structure. F: Cortex sections in pro-treated PTZ with GLE revealed mild to moderate neurofibrillary degeneration damaged neurons. G: Cortex sections in post treated PTZ with L-canitin, respectively, revealed a few numbers of damaged neurons with mild atrophy and diffuse vacuolar degeneration. H: Cortex sections in post treated PTZ with GLE revealed mild neuronal damage and atrophy. 

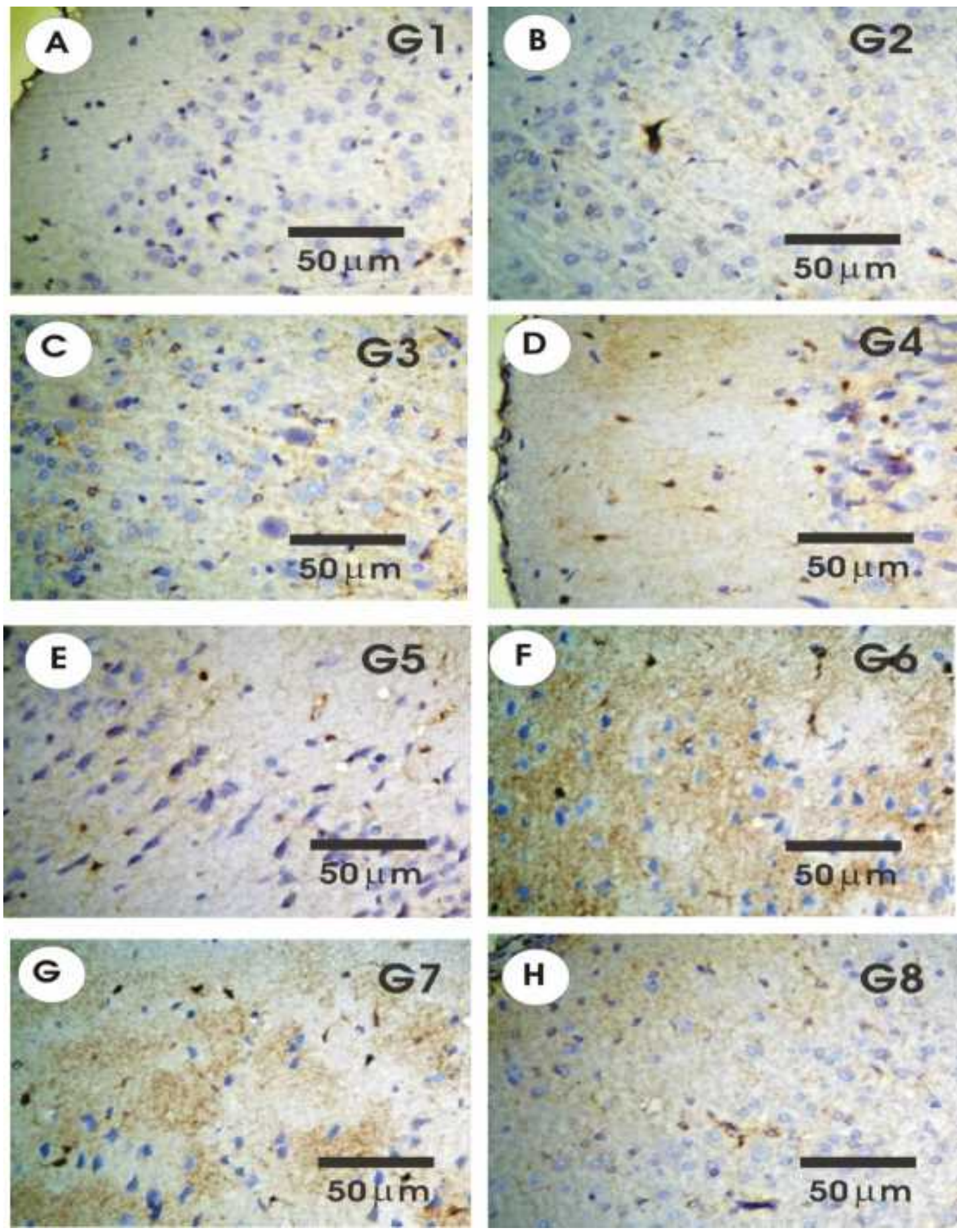

Figures 10A-10H:Photomicrographs of Ezerinimmunoreactivity (EZR-ir) in the coronal sections of rat cortex. A-C: Negative or Faint positive reaction for ERZ-ir in the control (A), L-carnitine(B) and GLE (C) groups, respectively. D:A moderate ERZ positive neurons in the cortex sections in PTZ rat group (G4). E\&F:Cortex coronal sections in both protreated PTZ with L-carnitine (G5) and with GLE (G6) revealed faint and mild positive reaction for ERZ-ir.G\&H:Cortex coronal sections in both post-treated PTZ with L-carnitine (G7) and with GLE (G8) revealed mild to moderate reaction for ERZ-ir. 
In this study, administration of pre and post treatment with L-carnitine induced significant increase in the GPX content.

This issimilar to nother studies which showed that Lcarnitine augmented the antioxidant defense capacity. This effect was mediated by an upregulation of antioxidant enzymes, an increase in the glutathione peroxidase levels (José et al., 2010).

Pre and post GBE induced significant increase in the GPX content. GBE can also enhance activities of antioxidant enzymes, such as superoxide dismutase (SOD), glutathione peroxidase (GPX) and catalase, thereby indirectly contributing as an antioxidant (Atmaca et al., 2005).

The present study demonstrated that a significant decrease in SOD levels was observed after PTZ administration. These results are in accordance with those ofSudha et al. (2001) and Rauca et al (2004) who showed marked reduction in antioxidant enzyme (SOD) in the PTZ-induced kindled group which leads to the production of free radicals and existence of oxidative stress in the brain of the kindled mice.

The present study demonstrating a significant increase in SOD levels was observed in pre and post treatment with GLE. These results are in accordance with those documented byAtmaca et al (2005) which showed that GLE can also enhance activities of antioxidant enzymes, such as superoxide dismutase (SOD). This indicates the antioxidant action of GLE.

The present study also demonstrated that a significant increase in SOD levels was observed in pre and post treatment with L-carnitine. These results are in accordance with the results ofLiJL (2012) who showed that L-carnitine enhances activities of superoxide dismutase (SOD).This indicates the influence of L-carnitine as an antioxidant for this enzyme.

The present study demonstrated that a significant decrease in catalase levels which were observed after PTZ administration. SenthilandRajkapoor (2010) reported that PTZ administration significantly decreased catalase activity in rate brain.

It was found that L-carnitine, pre and post treatments, was able to reverse action of PTZ on catalase activity and caused significant increase in the activity of catalase. Previous study by Cao et al. (2011) showed that Lcarnitine increase the activity of catalase, this indicate the ability of L-carnitine as a powerful antioxidant. Therefore, it may be useful as a supplementary therapy for chronic illnesses involving excessive oxidative stress.

Pre and post treatments with Ginkgo biloba were able to reverse action of PTZ against catalase activity and cause significant increase in the activity of catalase. Shaoping et al.(2011), showed that GLE cause significant increase in the activity of catalase. This GLE might scavenge the free radicals and enhance antioxidant enzyme activities.

The present study demonstrated that a significant decrease in GABA (the major inhibitory neurotransmitter in the brain) levels was observed after PTZ administration. This results are in agreement with those ofBikjdaouene (2004) who showed thatPTZ treatment decreased inhibitory amino acid glutamine levels in all brain areas.

Pre and post treatments with GLE were able to reverse action of PTZ against GABA and cause significant increase in the activity of GABA. This study agrees well with another one which suggests that the reported neuroprotective properties of GLE may, in part, be mediated through its ability to increase GABA-ergic, thus leading to an increase in the inhibitory effects of this neurotransmitter (Sasakiet al., 1999).

This study showed that pre and post treatments with Lcarnitine was able to reverse action of PTZ against GABA and cause significant increase in the activity of GABAand agrees well with the study undertaken by Sasaki et al., (1999) who suggested that the reported neuroprotective properties of L-carnitine may, in part, be mediated through its ability to increase GABA-ergic, thus leading to a increase in the inhibitory effects of this neurotransmitter.

The present study demonstrated that a significant increase in Glutamate (Glu) levels was observed after PTZ administration. These results are in agreement with those of Rasgadoet al. (2013) who showed that PTZ treatment increased excitatory amino acid glutamine levels in all brain areas.

In the present study pre and post treatment with GLE was able to reverse action of PTZ against Glu and cause significant decrease in the activity of GLU. The present study runs in line with another one which suggests that the reported neuroprotective properties of Ginkgo biloba may, in part, be mediated through its ability to reduce glutamate efflux, thus leading to a decrease in the excitotoxic effects of this neurotransmitter(Johns et al., 2002).

The current study indicated that pre and post treatments with L-carnitine induced significant decrease in the activity of GLU.Tastekin et al.,(2004), demonstrated that Lcarnitine protects against glutamate induced neurotoxicity. This protective effect of L-carnitine may occur via its antioxidant activity because free radical generation is a common result in glutamate induced neurotoxicity.

The present study demonstrated that a significant decrease in AChE levels was observed after PTZ administration. DeSales et al., (2010), showed increased acetylcholine content and decreased acetylcholinesterase activity in different brain regions during PTZ-induced seizures.

On the other hand, this study demonstrated that pre and post treatment L-carnitine induced significant increase in AChE. Packiasamy and Chinnakannu(2001) showed an increase in the avialability of $\mathrm{ACh}$ after L-carnitine supplementation and this might be responsible for the increase in the activity of AChE.

The present study demonstrated that pre and post treatments with Ginkgo bilobaleaf extract induced significant increase in AChE. Kehr et al.,(2012), showed that GLE causes an increase in the activity of AChE.

It can be concluded that pre and post-treatment pentylenetetrazole with Ginkgo biloba and L-carnitine 
prevented oxidative damage by inhibiting ROS production and improving antioxidant enzymes. This study suggests that neuroprotective properties of Ginkgo biloba and Lcarnitine through their ability to reduce glutamate efflux, thus leading to a decrease in the excitotoxic effects of this neurotransmitter.

\section{Referrences}

Abdel-Wahab B.A. andMetwally M.E. (2011): Ginkgo biloba Enhances the Anticonvulsant and Neuroprotective Effects of Sodium Valproate Against Kainic Acid-induced Seizures in Mice. Journal of Pharmacology andToxicology, 6: 679-690.

ADIS R and Profile D I (2003):EGb 761: Ginkgo biloba extract, Ginkor. Drugs in $\mathrm{R}$ and D. 4:188-93.

Aebi H. (1984): Catalase. In: Bergmeyer HU, (ed.) Methods of enzymatic analysis. New York: Academic Press;.p. 673-84.

Ahmed M F and Mahmoud M A. (2012): Effect of 1-carnitine on Pilocarpine induced seizures in rats. Menoufiya Medical Journal: 25: 1.

Akula, K.K., DhirA. And KulkarniS.K. (2007): Pro-convulsant effect of cefazolin sodium against pentylenetetrazol or Picrotoxin- induced convulsions in mice. Indian J. Exp. Biol., 45: 720-725.

Atmaca M., Tezcan E., Kuloglu M., Ustundag B. and Kirtas O. (2005): The effect of extract of ginkgo biloba addition to olanzapine on therapeutic effect and antioxidant enzyme levels in patients with schizophrenia. Psychiatry Clin Neurosci.;59(6):652-6.

Bancroft JD and Stevens GA (1990): Theory and Practice of Histological Techniques; 2nd Ed. Churchill Livingstone, London.

Beutler E., Duron O. and Kelly BM. (1963): Improved method for the determination of blood glutathione.J.Lab. Clin. Med;61: 882-8.

Bieber LL. (1988):Carnitine. Annu Rev Biochem. ;57:261-283.

Bikjdaouene L., Escames G., Camacho E., Leon J., Ferrer J.M., Espinosa A., Gallo M.A., De Dios Luna J. and Acuna-Castroviejo D. (2004): Effects of some synthetic kynurenines on brain amino acids and nitric oxide after pentylenetetrazole administration to rats. J. Pineal Res. 36, 267-277.

Cao Y., Qu HJ., Li P., Wang CB., Wang LX.and Han ZW. (2011): Single dose administration of L-carnitine improves antioxidant activities in healthy subjects. Tohoku J Exp Med.;224(3):209-13.

Cayir K., Karadeniz A., Yildirim A., Kalkan Y., Karakoc A .andKeles M. (2009): Protective effect of Lcarnitine against cisplatin-induced liver and kidney oxidant injury in rats. Cent Eur J Med; 4: 184-91.

Commission on Classification and Terminology of the International League Against Epilepsy. (1981): Proposal for revised clinical and electrographic classification of epileptic seizures. Epilepsia 22:489501 .
Corvino V., Marchese E., Michetti F. and Geloso MC. (2013): Neuroprotective strategies in hippocampal neurodegeneration induced by the neurotoxicanttrimethyltin.

Neurochem

Res.;38(2):240- 53.

de Sales Santos I. M., Feitosa C. M. and de Freitas R. M. (2010):Pilocarpineinduced seizures produce alterations on choline acetyltransferase and acetylcholinesterase activities and deficit memory in rats. Cell MolNeurobiol. 30(4):569-575.

Dhir A., Naidu P. S. and Kulkarni S. K. (2007):Neuroprotective effect of nimesulide, a preferential COX-2 inhibitor, against pentylenetetrazol (PTZ)-induced chemical kindling and associated biochemical parameters in mice. Seizure.,16(8):691-7.

Fisher RS., Acevedo C., Arzimanoglou A., Bogacz A., Cross H J., Elger CE., Engel J., Forsgren L.., French JA., Glynn M., Hesdorffer DC., Lee BI., Mathern GW., Moshe SL., Perucca E., Scheffer IE., Tomson T., Watanabe M. and WiebeS. (2014): A practical clinical definition of epilepsy. Epilepsia.;55 (4):475-482.

Fisher RS., van Emde Boas W., Blume W., Elger C., Genton P., Lee P. and Engel J Jr. (2005): Epileptic seizures and epilepsy: Defnitions proposed by the International League Against Epilepsy (ILAE) and the International Bureau for Epilepsy (IBE) Epilepsia. 2005;46(4):470-472.

Fossati P., et al., (1980):Clin. Chem. 26: 227-231. GodlevskiiLS.,Stepanenko KI., Lobasyuk BA., Sarakhan EV. andBobkova LM. (2004): The effects of electrical stimulation of the paleocerebellar cortex on penicillininduced convulsive activity in rats. NeurosciBehav Physiol.;34:797-802.

Gu" lc. (2006): in, Antioxidant and antiradical activities of 1-carnitine, Life Sci. 78:. 803-811.

Heba H. M. and Sameh S. T. (2012): Early treatment of radiation-induced heart damage in rats by caffeic acid phenethyl ester. European Journal of Pharmacology 692 : 46-51.

Helander TS., Carpén O., Turunen O., Kovanen PE., Vaheri A. and Timonen T. (1996): ICAM-2 redistributed by ezrin as a target for killer cells. Nature.;382:265-268.

Hermann B P., Jan Jones J E., Jackson D C. and Seidenberg M. (2012): Starting at the beginning: The neuropsychological status of children with new onset epilepsies. Epileptic Disord. 14(1): 12-21.

Johnston MV. (2007): Seizures in childhood. In: Kleigman RM, Behrman RE,Jenson HB, Stanton BP. Nelson Text Book of Pediatrics 18th Edition Philadelphia: Saunders Elsevier; p.2457-8.

Jones LL., McDonald DA. andBorum PR. (2010):Acylcarnitines: role in brain. Prog Lipid Res.;49:61-75.

José L., Miguel-Carrasco., Maria T.M., Afonso Mate.and Carmen M. V. (2010): Comparative effects of captoril and L-Carnitine on blood pressure and antioxidant enzyme gene expression in the heart of 
spontaneously hypertensive rats. European Journal of pharmacology. 632: 65-72.

Kehr J., Yoshitake S., Ijiri S., Koch .E, Noldner M. and Yoshitake T. (2012): Ginkgo biloba leaf extract (EG b 761® ) and its specific acylatedflavonol constituents increase dopamine and acetylcholine levels in the rat medial prefrontal cortex : possible implications for the cognitive enhancing properties of EGb $761 \circledR$. IntPsychogeriatr,; 24: 25- 34. 45: 325 .

Knedel, M., and R. Bottger.Klin.Wschr. (1967);

Kutluhan S., Naziroğlu M., Celik O. and Yilmaz M. (2009): Effects of selenium and topiramate on lipid peroxidation and antioxidant vitamin levels in blood of pentylentetrazol-induced epileptic rats. Biol. Trace Elem. Res.;129:181-189.

Laskin J. D., Black A. T., Jan Y. H., Sinko P. J., Heindel N. D., Sunil V., Heck D. E. and Laskin D. L. (2010): Oxidants and antioxidants in sulfur mustardinduced injury: Ann N Y AcadSci, v. 1203, p. 92-100.

Li JL., Wang QY., Luan HY., Kang ZC.And Wang CB. (2012):Effects of L-carnitine against oxidative stress in human hepatocytes: involvement of peroxisome proliferator-activated receptor alpha. J Biomed Sci. $21 ; 19-32$.

Lindroth P. and Mopper K. (1979): High performance liquid chromatographic determination of sub picomole amounts of amino acids by precolumn fluorescence derivatization with o-phthal-dialdehyde, Analyt. Chem., 51: 1667-1674.

Liu M., Sheng Z., Cai L., Zhao K., Tian Y. and Fei J. (2012): Neuronal conditional knockout of NRSF decreases vulnerability to seizures induced by pentylenetetrazol in mice.ActaBiochimBiophys Sin (Shanghai) 44:476-482.

Liu S. Q., Yu J. P., Chen H. L., Luo H. S., Chen S. M. and Yu H. G. (2006): "Therapeutic effects and molecular mechanisms of Ginkgo biloba Extract on liver fibrosis in rats," The American Journal of Chinese Medicine, vol. 34, no. 1, pp. 99-114.

Mandhane S. N., Aavula K.and Rajamannar T. (2007): Timedpentylenetetrazol infusion test: a comparative analysis with s.c.PTZ and MES models of anticonvulsant screening in mice. 16(7):636-44.

Martinotti G., Andreoli S., Reina D., Di Nicola M., Ortolani I. and Tedeschi D. (2011): Acetyl-1Carnitine in the treatment of anhedonia, melancholic and negative symptoms in alcohol dependent subjects. Prog.Neuropsychopharmacol.Biol

Psychiatry 2011, 35(4):953-958.

Moreno S., Carvalho J. J., Nascimento A. L. R., Freitas R. S., Diré G. F., Lima E. A., Lima-Filho G. L., Rocha E. K. and Bernardo-Filho M. (2004):Biodistribution of sodium pertechnetate and light microscopy of organs isolated from the rats: Study of the effects of a Ginkgo biloba extract. Pak. J. Nutr., 3:64-7.

$\begin{array}{llll}\text { Nalecz } & \text { K. A., Miecz } & \text { D., Berezowski }\end{array}$ V. and Cecchelli R. (2004): Carnitine: transport and physiological functions in the brain. Mol. Aspects Med. 25, 551-567.

Nieoczym D., AlberaE.and Kankofer M., (2008): Maximal electroshock induces changes in some markers of oxidative stress in mice. J Neural Transm., 115:19-25.

Nishikimi M., Roa N.A. and Yogi K. (1972): Biochem.Bioph. Res. Common., 46: 849-854.

Ohkawa H., Ohishi W. and YagiK. (1979). Anal.Biochem .95: 351.

PackiasamyJ A R. and Chinnakannu P. (2001): Protective Efficacy of L-Carnitine on Acetylcholinesterase Activity in Aged Rat Brain. Journal of Gerontology: Biological Sciences. 56( 3): 140-141.

Paglia D.E. and Valentine W.N. (1967): J. Lab. Clin. Med. 70: 158-169.

Patsoukis N., Zervoudakis G., Georgiou C.D., Angelatou F., Matsokis N.A. and Panagopoulos N.T. (2004):Thiol redox state and lipid and protein oxidation in the mouse striatum after pentylenetetrazol-induced epileptic seizure. Neuroscience Letters 357 : 83-86.

Pehlivan M., Coşkun A., Zengin A., Aslaner A. and Yavuz T. (2009): Does L-carnitine increase serum TNF $-\alpha$ and IGF-1 during liver regeneration in the rat? Turk $\mathrm{J}$ Med Sci; 39: 875-80.

Pinakinik S., Vasanth K. and Namita R. (2005): Evaluation of Antidiabetic Activity of Ginkgo Biloba in Streptozotocin Induced Diabetic Rats. Iranian Journal of Pharmacology \& Therapeutics.4(1): 16-19.

Rasgado L A V., Reyes G C. and Díaz F V. (2013): Effect of convulsant drugs in GDH activity and oxygen consumption in mouse brain. Journal of Medicine and Medical Sciences. 4(1) pp. 34-42.

Rauca C., Wiswedel I., Zerbe R., Keilhoff G. and Krug M. (2004): The role of superoxide dismutase and alpha-tocopherol in the development of seizures and kindling induced by pentylenetetrazol - influence of the radical scavenger alpha-phenyl-N-tert-butyl nitrone. Brain Res.;1009:203-212.

Rodriguez de Turco E. B., Droy-Lefaix M. T., and Bazan N. G. (1993): Decreased electroconvulsive shockinduced diacylglycerols and free fatty acid accumulation in the rat brain by Ginkgo biloba extract (EGb 761): selective effect in hippocampus as compared with cerebral cortex. J. Neurochem. 61, 1438-1444.

Salama A. F., Kasem S. M., Tousson E. and Elsisy M. K. (2012): Protective role of L-carnitine and vitamin $\mathrm{E}$ on the kidney of atherosclerotic rats. Biomedicine \& Aging Pathology 2012;2: 212-5.

Salama A., Kasem S., Tousson E. and Elsisy MK. (2013): L-carnitine and vitamin E alleviate reproductive toxicity caused by triton WR 1339 in male albino rats. Toxicology and Industrial Health;2013. DOI: 10.1177/0748233712472523.

Samuel N., Finkelstein Y., SingerS R. and Oberbaum M. (2008): Herbal medicine and epilepsy: Proconvulsive effects and interactions with antiepileptic drugs. Epilepsia 49(3): 373-380. 
Sarkisian MR. (2001): Overview of the Current Animal Models for Human Seizure and Epileptic Disorders.EpilepsyBehav. 2:201-16.

Sasaki K., Hatta S., Haga M. and Ohshika H. (1999): Effects of bilobalide on g-aminobutyric acid levels and glutamic acid decarboxylase in mouse brain. Eur $\mathrm{J}$ Pharmacol 367: 165-173.

Satoh K. (1978): ClinicaChimicaActa .90:37.

SekarBabu M., Sangeetha G., Balammal G. and Mohan Krishna L. (2011): Effect of Ageratum Conyzoides L. Extracts on antioxidant enzymes levels in rat brain after induced of seizures. International Journal of Pharmacology \& Toxicology / 1(1), 27-31.

SenthilKumar K.K. and Rajkapoor B. (2010): Study on phytochemical profile and anti-epileptic activity of oxalis corniculata 1. International Journal of Biological \& Pharmaceutical Research, 1(1), 33-36.

ShankaranarayanaraoBS.,Raiu TR. and Meti BL. (1998): Self stimulation of lateral hypothalamus and ventral tegmentum increases the levels of noradrenaline, dopamine, glutamate and AChE activity, but not 5-hydroxyl tryptamine and GABA levels in hippocampus and motor cortex. Neurochem. Res., 23(8): 1053-1059.

Shaoping Lu., Xia Guo. and Pinting Zhao. (2011) Effect of Ginkgo Biloba Extract 50 on Immunity and Antioxidant Enzyme Activities in Ischemia Reperfusion Rats. Molecules., 16(11), 9194-9206.
Silva-Adaya D., Perez-De La Cruz V. and Herrera-Mundo MN. (2008):Excitotoxic damage, disrupted energy metabolism, and oxidative stress in the rat brain: antioxidant and neuroprotective effects of L-carnitine. J Neurochem;105:677-89.

Sudha K., Rao AV. and Rao A. (2001): Oxidative stress and antioxidants in epilepsy. Clin. Chem., 303: 1924.

Tastekin A., Gepdiremen A., Ors R., Emin BM. and Halici Z. (2005): L-Carnitine protects against glutamate- and kainic acid-induced neurotoxicity in cerebellar granular cell culture of rats. Brain Dev.;27:570573.

Tousson, E., Hafez E., Zaki S., and Gad A. (2014): P53, Bcl-2 and CD68 expression in response to amethopterin-induced lung injury and ameliorating role of 1-carnitine: Biomed Pharmacother, v. 68, p. 631-9.

Waggas A. and Al-Hasani R. (2010): Neurophysiological study on possible protective and therapeutic effects of Sidr (Zizyphusspina-christi) leaf extract in male albino rats treated with pentylenetetrazol. Saudi Journal of Biological Sciences, 17(4): 269- 274.

Wu C., Gopal K., Gross GW., Lukas TJ. and Moore EJ. (2011): An in vitro model for testing drugs to treat tinnitus. Eur J Pharmacol 667:188-194. 